\title{
Novel multiplex real-time quantitative PCR detecting system approach for direct detection of Candida auris and its relatives in spiked serum samples
}

\author{
Amir Arastehfar ${ }^{\ddagger}, 1$, Wenjie Fang ${ }^{\ddagger 1,2,3}$, Farnaz Daneshnia ${ }^{1}$, Abdullah MS Al-Hatmi ${ }^{1,4,5}$, \\ Wanqing Liao ${ }^{2,3}$, Weihua Pan*,2,3, Ziauddin Khan ${ }^{6}$, Suhail Ahmad ${ }^{6}$, Katharina Rosam \\ Michaela Lackner ${ }^{7}$, Cornelia Lass-Flörl ${ }^{7}$, Ferry Hagen ${ }^{1}$ \& Teun Boekhout**,1,3,8 \\ ${ }^{1}$ Westerdijk Fungal Biodiversity Institute, Utrecht 3584, The Netherlands \\ 2Department of Dermatology, Shanghai Changzheng Hospital, Second Military Medical University, Shanghai 200003, PR China \\ ${ }^{3}$ Shanghai Key Laboratory of Molecular Medical Mycology, Shanghai Institute of Mycology, Shanghai Changzheng Hospital, \\ Second Military Medical University, Shanghai 200003, PR China \\ ${ }^{4}$ Center of Expertise in Mycology Radboud University Medical Center, Canisius Wilhelmina Hospital, Nijmegen 6500HB, The \\ Netherlands \\ ${ }^{5}$ Ministry of Health, Directorate General of Health Services, PO Box 393, 100 Muscat, Oman \\ ${ }^{6}$ Department of Microbiology, Faculty of Medicine, Kuwait University, Safat, Kuwait \\ ${ }^{7}$ Division of Hygiene \& Medical Microbiology, Medical University of Innsbruck, Innsbruck, Austria \\ ${ }^{8}$ Institute of Biodiversity \& Ecosystem Dynamics, University of Amsterdam, Amsterdam 1012 WX, The Netherlands \\ *Author for correspondence: Tel.: +86 8188 5494; panweihua@smmu.edu.cn \\ **Author for correspondence: Tel.: +31 62929 0360; t.boekhout@westerdijkinstitute.nl \\ ${ }^{\ddagger}$ Authors contributed equally
}

The multidrug-resistant opportunistic yeast species of Candida auris, Candida haemulonii, Candida duobushaemulonii and Candida pseudohaemulonii continue to endanger the healthcare settings around the globe. Due to the lack of a specific QPCR assay for detection of these species from clinical samples, we developed a multiplex qPCR assay. Analytical specificity and sensitivity showed $100 \%$ specificity and the sensitivity of up to ten genomes of target species with a high value of reproducibility $\left(R^{2}>0.99\right)$. Subsequently, from spiked serum samples, our qPCR specifically could detect up to ten genomes of $C$. auris and one genome of C. haemulonii, C. duobushaemulonii and C. pseudohaemulonii $\left(R^{2}>0.98\right)$. Lack of cross reaction with the human DNA, a high degree of specificity and sensitivity, showed the potential of our multiplex PCR for direct detection of $C$. auris and closely related species from serum samples of suspected patients. Future studies are warranted to assure its applicability in clinical settings.

First draft submitted: 11 August 2018; Accepted for publication: 20 November 2018; Published online: 12 December 2018

Keywords: bloodstream infection $\bullet$ Candida auris $\bullet$ melting curve analysis $\bullet$ qPCR

Nowadays, controlling infections caused by opportunistic yeast species is challenged owing to increasing of immunocompromised population, emerging pathogenic species and increasing prevalence of antifungal-resistant isolates, which potentially lead to diagnostic and therapeutic failures and increased healthcare costs $[1-4]$. The globally fast-spreading Candida auris currently is regarded as one of the most important opportunistic emerging yeast species, which caused a huge concern for healthcare settings [5-7]. Using routine typing protocols, clinical isolates of $C$. auris are clustered in four distinctive clades namely, east Asian, south Asian, South American and African [8]. From 2012 to 2017, > 361 isolates of C. auris were obtained from blood cultures, accounting for $67.48 \%$ of the all recovered isolates from clinical samples, worldwide [9]. On the other hand, the other closely related species of C. auris namely, Candida haemulonii, Candida duobushaemuloni and Candida pseudohaemulonii have been reported to possess a multidrug-resistant profile and all have been isolated from blood samples in various countries [10-12]. Candida haemulonii, which was first known as a human pathogen in 1984, is being increasingly reported worldwide [6,10]. Additionally, recent surveillance studies of candidemia conducted in Panama, revealed 
Table 1. Primers and targets used in the qPCR assay.

\begin{tabular}{|c|c|c|c|c|}
\hline Target species & Primer name & Primer sequence & Target loci & Product size (bps) \\
\hline \multirow[t]{2}{*}{ Candida auris } & Auris-F & CGGTGGCGTTGCATTCA & Internal Transcribed Spacer 2 & 244 \\
\hline & Auris-R & CTGCTGCTAGAACACCTCGT & 285 rDNA & \\
\hline \multirow[t]{2}{*}{ Candida haemulonii } & Haemu-F & CCGAGGACCGCAGCAAT & $28 \mathrm{~S}$ rDNA & 181 \\
\hline & Haemu-R & CCCGTCCGAGCACTTCCA & $28 \mathrm{~S}$ rDNA & \\
\hline \multirow[t]{2}{*}{ Candida duobushaemulonii } & Duobu-F & GAACGCACATTGCGCCTTGG & $5.8 \mathrm{~s}$ rDNA & 115 \\
\hline & Duobu-R & GTAGACTTCGCTGCGGATATGTTA & Internal Transcribed Spacer 2 & \\
\hline \multirow[t]{2}{*}{ Candida pseudohaemulonii } & Pseud-F & TAACCTGCGGAAGGATCATTAAG & 185 rDNA & 197 \\
\hline & Pseud-R & AATGTGCGTTCAAAGATTCGATG & $5.8 \mathrm{~S}$ rDNA & \\
\hline
\end{tabular}

that almost half of 36 isolates primarily identified as $C$. auris were $C$. duobushaemulonii [13], which highlights the importance of correct identification of other closely related species of $C$. auris. Candida pseudohaemulonii that for the first time in 2006 was detected in the blood sample of a Thai patient showing resistance to amphotericin B and azoles [14], was accountable for approximately a third of clinical isolates identified as $C$. haemulonii in a Korean surveillance study and surprisingly all of the isolates were from blood samples [10].

For these multidrug-resistant pathogens, rapid and precise diagnosis is important to initiate appropriate antifungal treatment, prognosis improvement and outbreak control [15-17]. Up to now, $>20$ identification methods have been published for $C$. auris $[9,18-20]$. Biochemical assays as one of the most widely used phenotypic approaches, besides of being time consuming and expensive, cannot properly identify these species [21]. Recently, several species-specific PCRs have been established to identify $C$. auris from DNA samples derived from pure colonies or environmental swab samples $[18,19]$. Although the need for a culture-independent molecular method is urgent, unfortunately, this is still lingering as an unsolved issue.

Herein, we developed the first multiplex qPCR assay for $C$. auris, and its relatives, that is, $C$. haemulonii, $C$. duobushaemulonii and $C$. pseudohaemulonii, which can detect these opportunistic yeast species directly from spiked serum samples with a clinically relevant limit of detection (LOD).

\section{Materials \& methods}

Fungal strains

Two hundred and eighty-two reference strains and clinical isolates were used in two different analytical validation test sets. The first test set included 177 reference strains consisted of 33 C. auris, 25 C. haemulonii, five $C$. duobushaemulonii, three C. pseudohaemulonii and 111 fungal strains representing 68 other closely or distantly related species, which were included for testing the analytical sensitivity, reproducibility, specificity and proficiency testing (Supplementary Tables $1 \& 2$ ). Reference strains were obtained from Westerdijk Fungal Biodiversity Institute, Utrecht, Netherlands. Human genomic DNA (male, $10 \mathrm{ng} / \mu \mathrm{l}$, Thermo Fisher Scientific, MA, USA) was also used for specificity testing. The second test set included 105 clinical isolates obtained from Faculty of Medicine, Kuwait University, Kuwait, which were previously identified as $C$. auris by matrix-assisted laser desorption/ionization TOF mass spectrometry (MALDI-TOF MS; Bruker Corporation, MA, USA) [22,23].

\section{Primer design}

Sequences of rDNA for $C$. auris (including the isolates of the four clades analyzed by Lockhart et al. in 2016 [8] and more isolates after 2016) and its relatives were retrieved from GenBank (www.ncbi.nlm.nih.gov/genbank/) for primer design. We analyzed the rDNA region of 233 global isolates for the reverse primer of $C$. auris, of which 138 of the sequences were from our own database and the rest were from Genbank. Only primers meeting the following criteria were chosen for further optimization: they should cover all the clades of target species; no cross reaction with other clinically and environmentally important fungi and human DNA; production of a single discriminative melting peak for each locus, which is at least $1^{\circ} \mathrm{C}$ different from the other locus; and being compatible with the rest of primers in the same reaction in terms of primer $\mathrm{Tm}$ values and primer homo/heterodimer formations. uMELT (www.dna.utah.edu/umelt/umelt.html) was used to predict PCR products melting temperature. IDT OligoAnalyzer 3.1 (https://eu.idtdna.com/calc/analyzer) was used to predict primer Tm values and homo/heterodimers. Subsequently, oligonucleotides were synthesized by Integrated DNA Technology Company (IDT, Leuven, Belgium). Table 1 shows the primers information used in this study. 

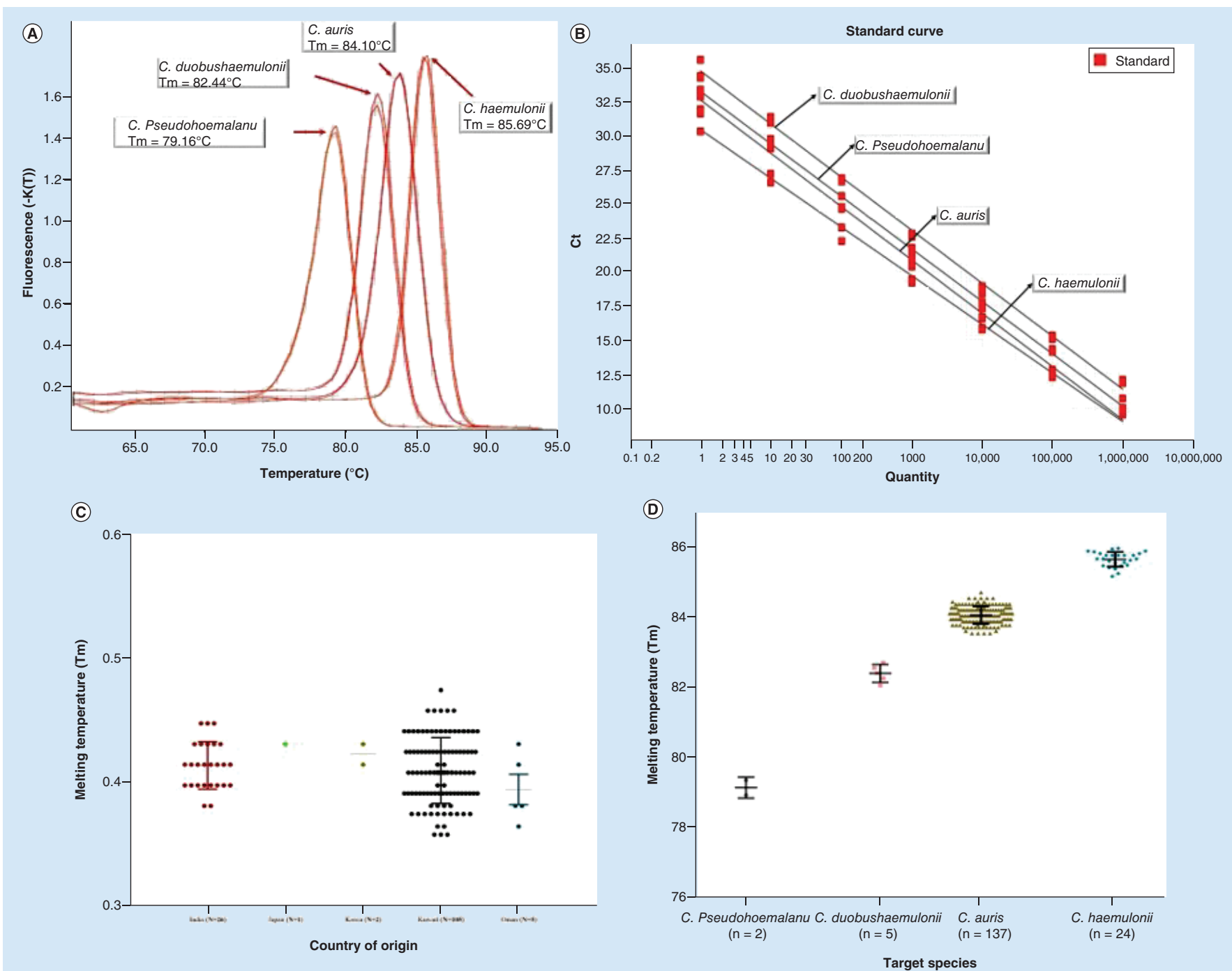

Figure 1. Shows the analytical features obtained for our qPCR assay. (A) Melting profile of target species including Candida pseudohaemulonii, Candida duobushaemulonii, Candida auris and Candida haemulonii. (B) Standard curves of four target species over seven orders of magnitude. (C) Melting temperature distribution of C. auris in five countries. (D) Shows the Tm distribution of four target species.

\section{Analytical validation}

Log-10 serial dilutions (starting from $1 \mathrm{ng} / \mu \mathrm{l}$ ) of DNA of all four target species were prepared to find the LOD and the reproducibility of our assay. The analytical specificity was evaluated using $1 \mathrm{ng} / \mu \mathrm{l}$ DNA of diverse range of other yeast species, filamentous fungi and human.

Two technicians ran the proficiency testing in a blind fashion using DNA of eight target strains of C. auris, $C$. pseudohaemulonii, $C$. duobushaemulonii and $C$. haemulonii dispersed among 111 nontarget strains representing 68 fungal species (Supplementary Table 3). One technician, who was aware of the identity of each DNA samples, randomly distributed them and provided the second technician with the blinded test set. Besides, single pure colonies of $C$. auris, $C$. pseudohaemulonii, $C$. duobushaemulonii and $C$. haemulonii were subjected to our multiplex PCR.

\section{Institutional ethical committee approval}

As clinical validation phase of our study required serum samples, the procedure of serum sampling was reviewed and approved by the ethical committee members of Medical University of Innsbruck. 

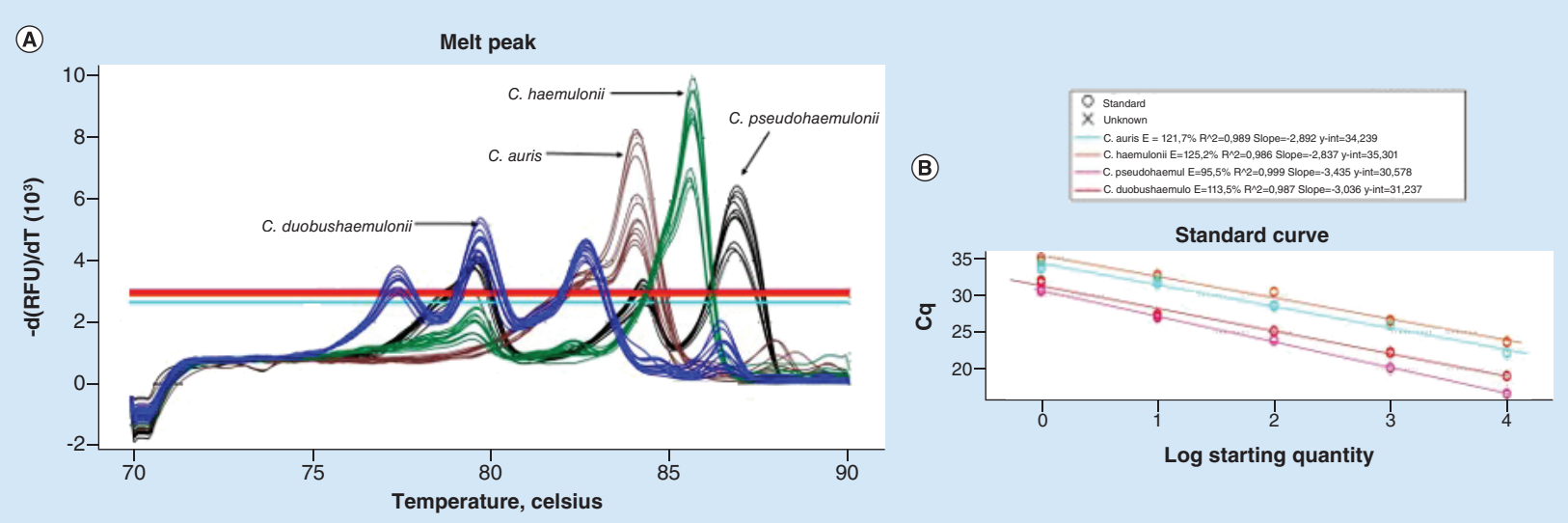

Comparison of $\mathrm{Ct}$ values obtained for spiked serum and pure DNA samples

(c)

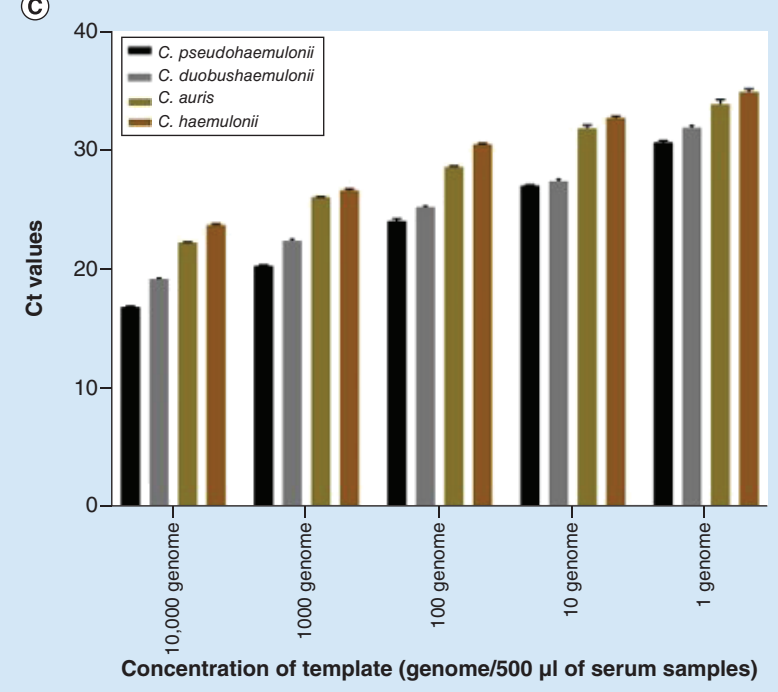

(D) Melt peak

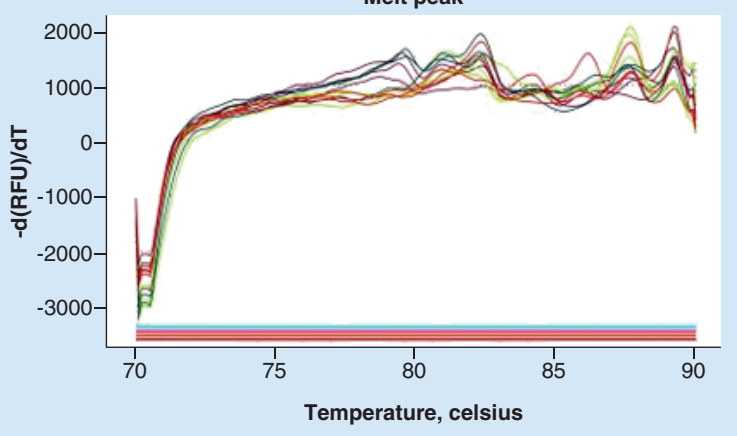

Figure 2. Analytical information derived from spiked serum samples. (A) Serum samples spiked with five orders of magnitude $\left(10^{4}-10^{0}\right)$ of the DNA of target species. (B) The efficiency of target species derived from spiked serum samples $\left(R^{2}>0.98\right)$. (C) Ct values of spiked serum samples with five order of magnitude of DNA $\left(10^{4}-10^{\circ}\right)$ of four target species. (D) And, the irregular-shaped melting curves obtained from negative serum samples. The data obtained from the standard curves including efficiency, limit of detection and R2 values are automatically calculated by the software installed on the real-time PCR machine and they were not measured manually.

\section{Spiked serum samples}

Due to the lack of serum samples of suspected or proven cases, we proceeded with spiking serum samples with the DNA samples obtained from C. auris (CBS 10913), C. haemulonii (CBS 5149), C. duobushaemulonii (CBS 7798) and C. pseudohaemulonii (CBS 10004).

For serum preparation, blood clots were subjected to centrifugation with $3000 \times g$ for $15 \mathrm{~min}$. Serum samples were distributed in $500 \mu \mathrm{l}$ aliquots in DNase/RNase sterile $2 \mathrm{ml}$ tubes and stored at $-20^{\circ} \mathrm{C}$ for future uses. Serum samples were collected from two donors.

Five hundred microliters of serum samples were spiked with serially diluted DNA samples (from $10^{4}$ genome to $10^{0}$ genome) obtained from C. auris (CBS10913), C. haemulonii (CBS5149), C. duobushaemulonii (CBS7798) and C. pseudohaemulonii (CBS10004). As, $100 \mathrm{pg}$ of DNA of C. albicans estimated to be $10^{4}$ genomes [24] and due to the similarity of genome size of the $C$. albicans $(14.6 \mathrm{Mb}$ ) with those of $C$. auris $(12.36 \mathrm{Mb}), C$. haemulonii (13.6 Mb), C. duobushaemulonii (12.58 Mb) and C. pseudohaemulonii (12.64 Mb), we considered $1 \mathrm{ng}$ DNA of target species as $10^{5}$ genomes. 
(A) Melt peak

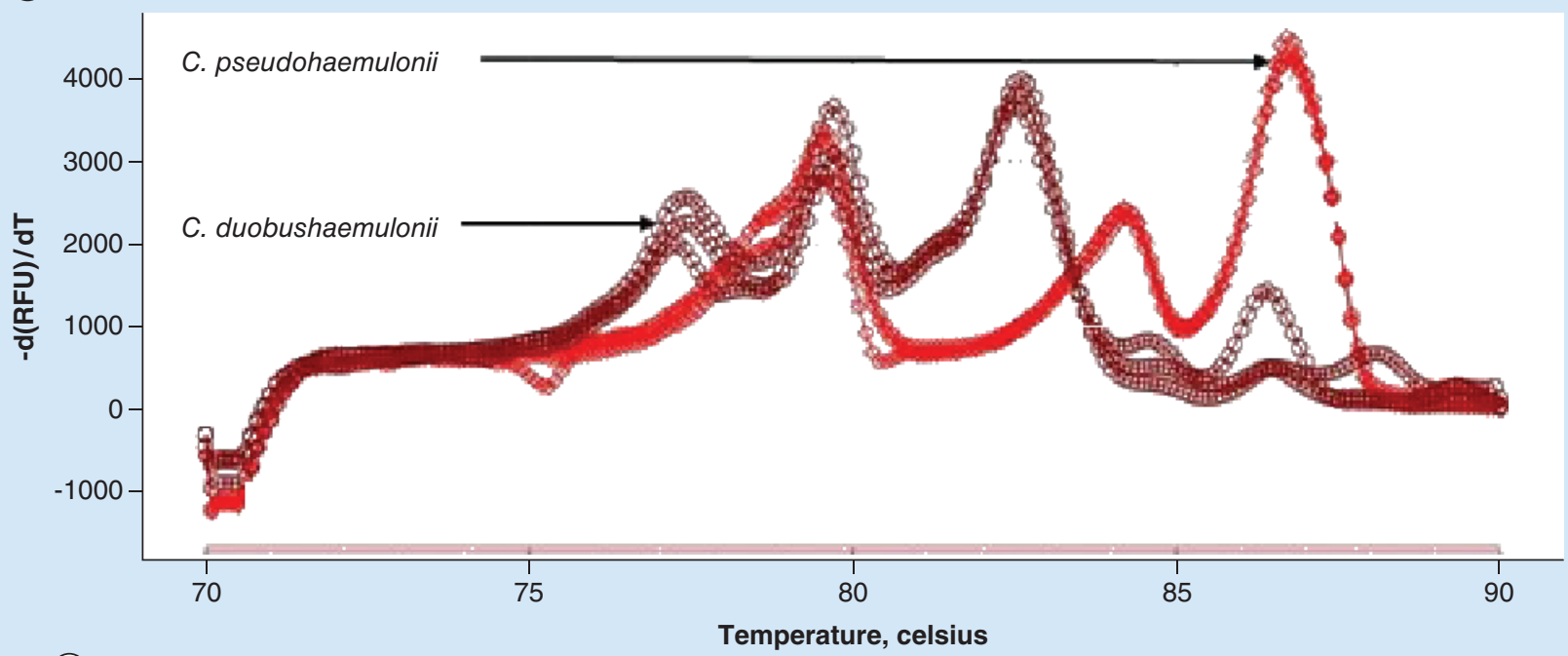

(B)

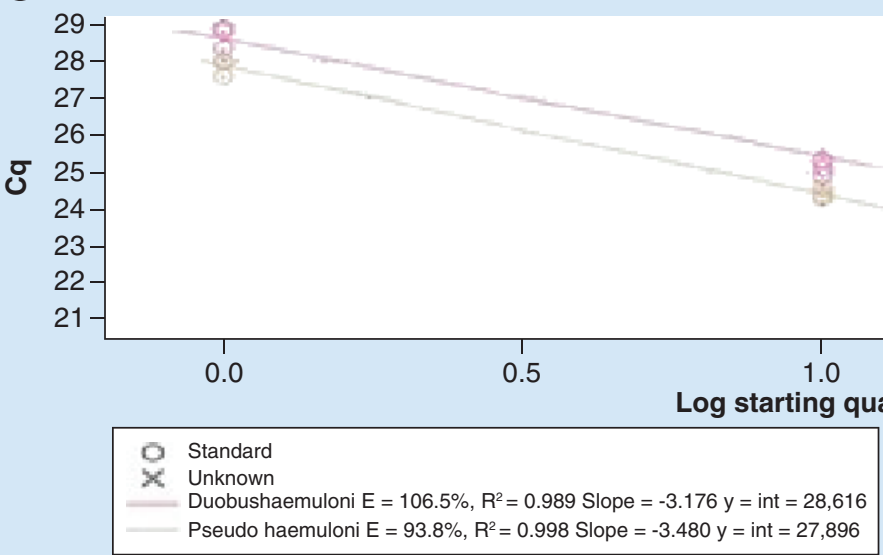

(C)

Melt peak

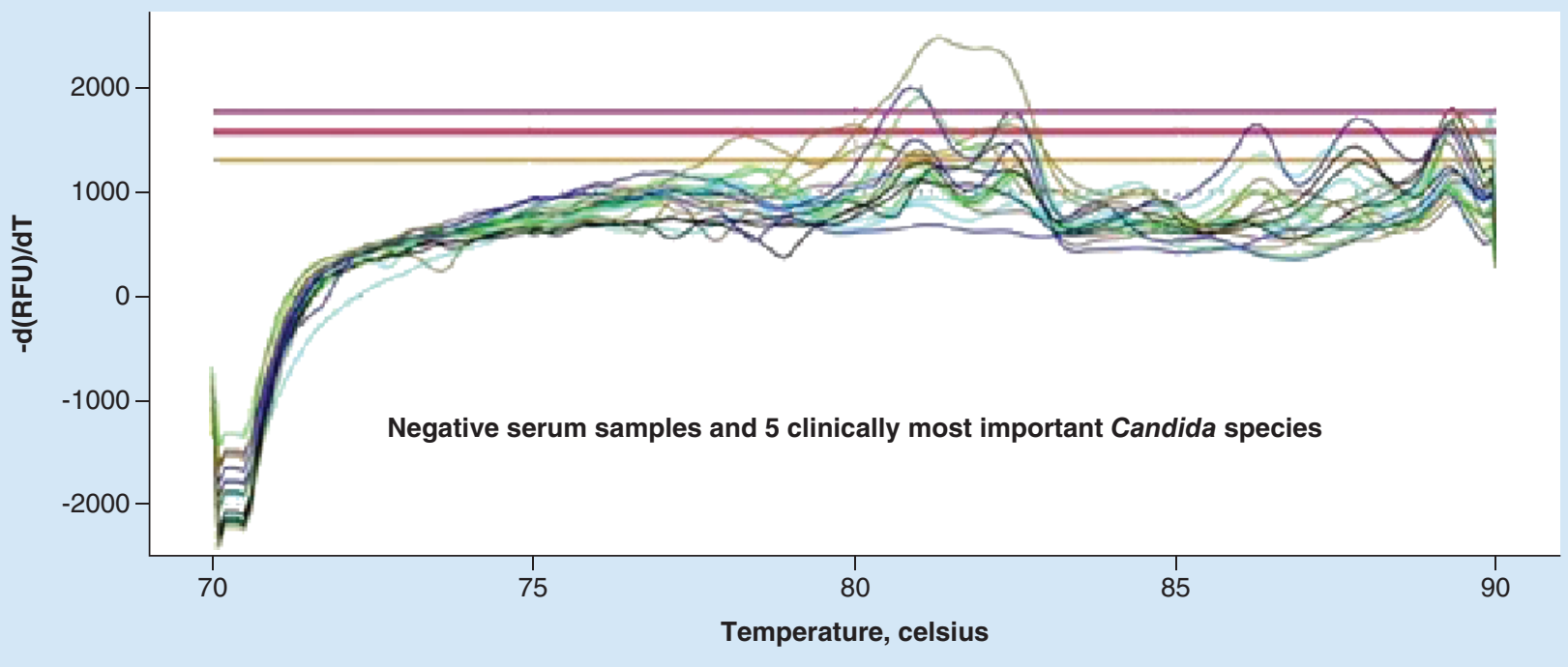

Figure 3. Melting curve analysis for Candida duobushaemulonii and Candida pseudohaemulonii (102-100 genomes) and negative samples. Shows the melting curve obtained from spiked serum samples with DNA $\left(10^{2}-10^{0}\right.$ genomes) of Candida duobushaemulonii and Candida pseudohaemulonii (A \& B), and Candida albicans, Candida parapsilosis, Candida glabrata, Candida tropicalis, Candida krusei and negative serum samples (C). As it is evident from the figure, nontarget Candida species and negative serum samples (C) generated unusual melt curves that are readily distinguishable from those of target species (A). Although, normal standard curves include at least four concentration points, due to the clinical concentration relevance, only three concentration points (1, 10 and 100 genomes) were used. 


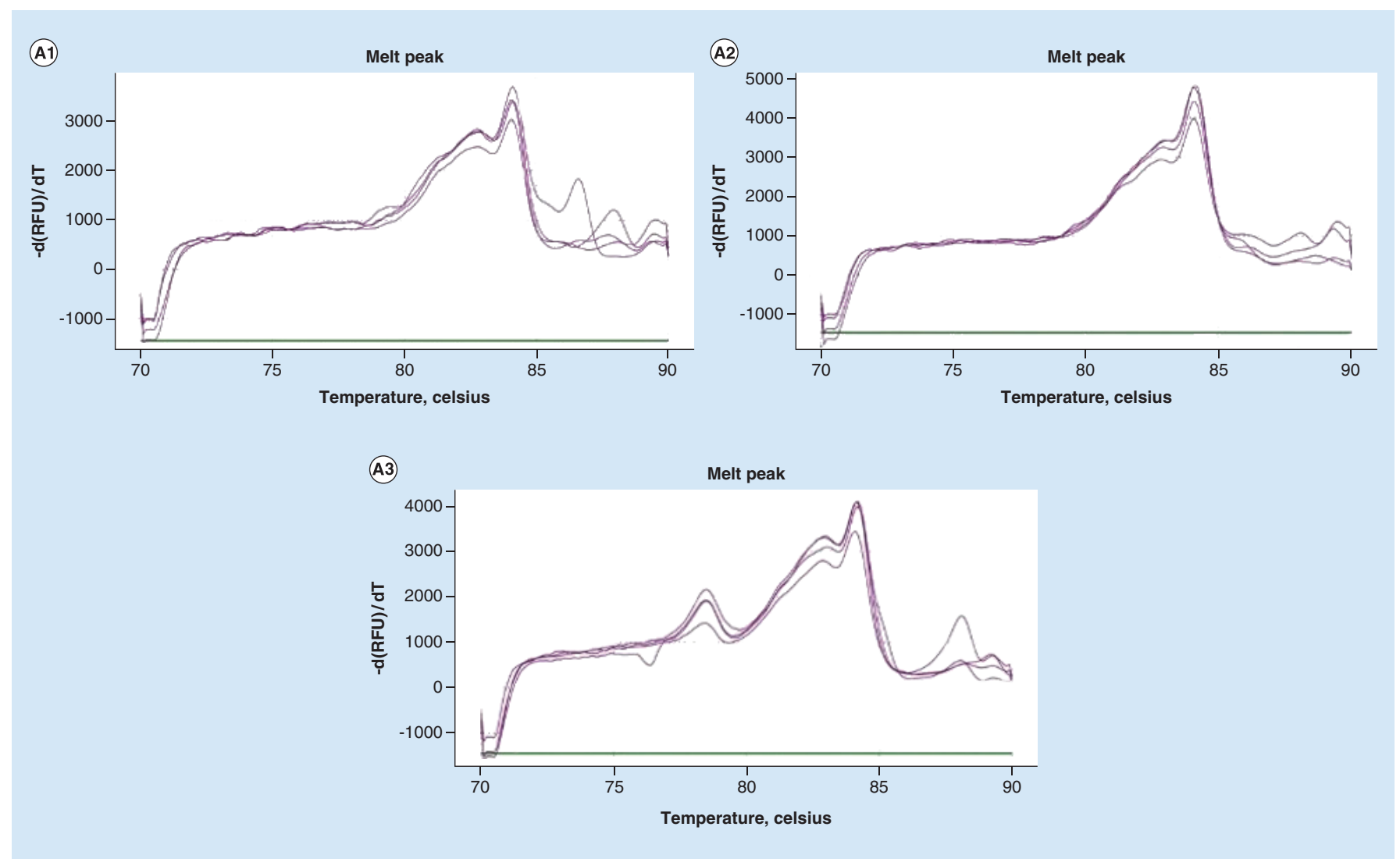

Figure 4. Melt curves obtained from serum samples spiked with mixture of Candida albicans and each target species, individually. (A1) A total of 100 genome (Ge) of Candida albicans-10 Ge of Candida auris; (A2) $100 \mathrm{Ge}$ of C. auris-10 Ge of C. albicans; (A3) $10 \mathrm{Ge}$ of C. auris-10 Ge of C. albicans; (B1) $100 \mathrm{Ge}$ of C. albicans-10 Ge of Candida duobushaemulonii; (B2) $100 \mathrm{Ge}$ of C. duobushaemulonii-10 Ge of C. albicans; (B3) $10 \mathrm{Ge}$ of C. duobushaemulonii-10 Ge of C. albicans; (C1) $100 \mathrm{Ge}$ of C. albicans-10 Ge of Candida haemulonii; (C2) $100 \mathrm{Ge}$ of C. haemulonii-10 Ge of C. albicans; (C3) $10 \mathrm{Ge}$ of C. haemulonii-10 Ge of C. albicans; (D1) $100 \mathrm{Ge}$ of C. albicans-10 Ge of Candida pseudohaemulonii; (D2) $100 \mathrm{Ge}$ of C. pseudohaemulonii-10 Ge of C. albicans; (D3) $10 \mathrm{Ge}$ of C. pseudohaemulonii-10 Ge of C. albicans.

\section{Simulating mixed infection in spiked serum samples containing the DNA of C. albicans \& target species}

As C. albicans shown to be the most predominant isolated species in coinfection cases [25], hence DNA sample of each of target species (100 and 10 genomes) were individually mixed with DNA samples of C. albicans (100 and 10 genomes as follows, 100 genome of $C$. albicans -10 genome of target species, 100 genome of target species-10 genome of $C$. albicans, 10 genome of $C$. albicans -10 genome of target species). Twelve serum samples (500 $\mu \mathrm{l})$ were spiked with the DNA mixtures and the serum samples were subjected to DNA extraction as mentioned in the following.

\section{DNA extraction from fungal cells \& simulated serum samples}

DNA extraction from pure colonies was performed by cetyltrimethyl ammonium bromide (CTAB) method [26]. The obtained DNA samples were checked for purity by NanoDrop ${ }^{T M} 2000$ (Thermo Fisher Scientific) and for quantity by QuBit dsDNA BR Assay Kit (Thermo Fisher Scientific Corporation). All the DNA samples were standardized to $1 \mathrm{ng} / \mu \mathrm{l}$ for further checking of sensitivity and specificity.

Serum samples were extracted by QIAamp ${ }^{\circledR}$ UltraSens ${ }^{\circledR}$ Virus kit (QIAGEN, Hilden, Germany) with a slight modification of the manufacturer's protocol. In the last step of elution of DNA samples, instead of two continuous elution stages with $30 \mu \mathrm{l}$, the DNA samples were eluted by two steps of 25 and $20 \mu \mathrm{l}$. In order to monitor the phenomenon of cross contamination during DNA extraction steps, negative serum samples (without DNA of target species) were included. Eight microliters of each extracted DNA samples were run in the qPCR assays in duplicate. 


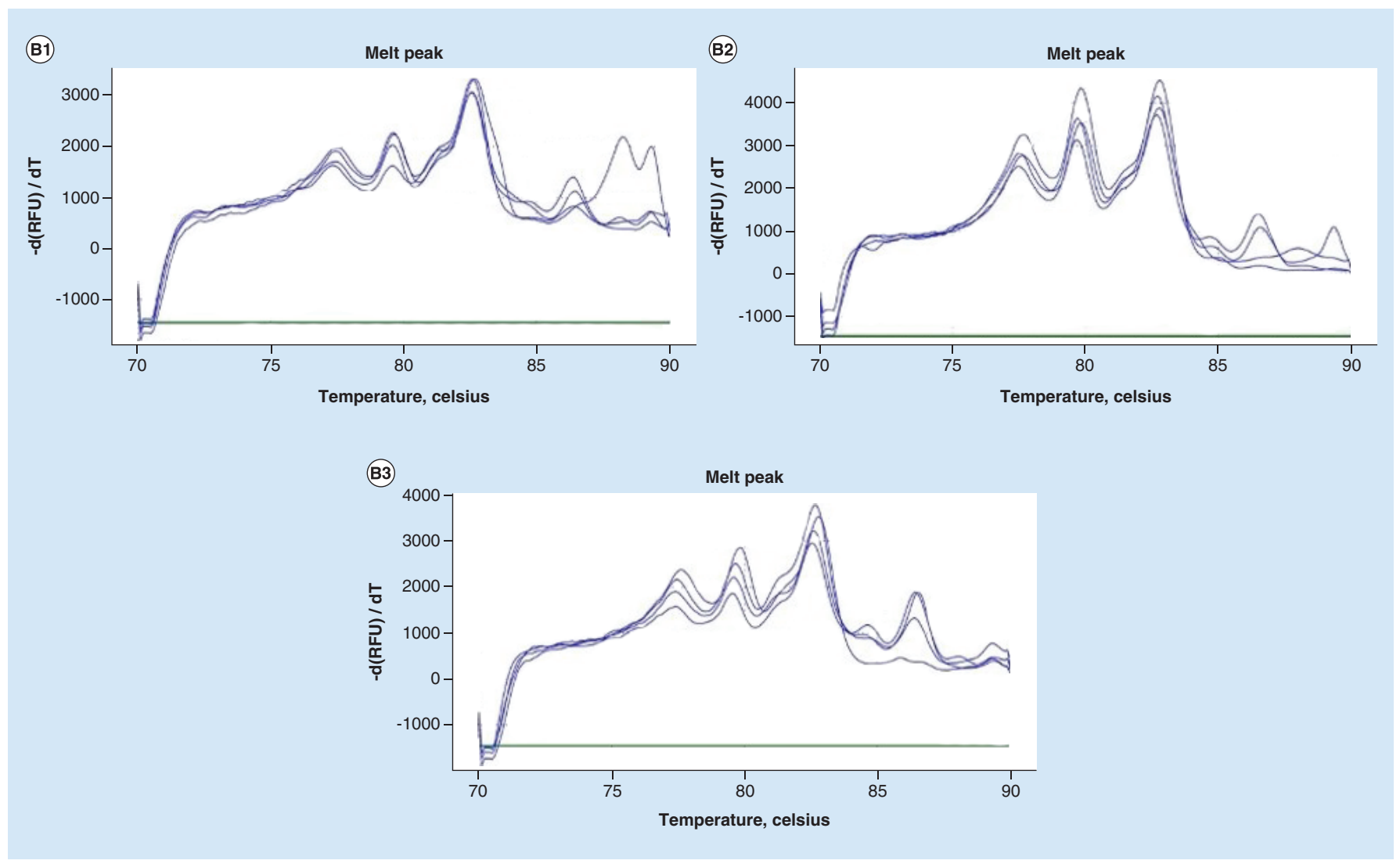

Figure 4. Melt curves obtained from serum samples spiked with mixture of Candida albicans and each target species, individually (cont.). (A1) A total of 100 genome (Ge) of Candida albicans-10 Ge of Candida auris; (A2) $100 \mathrm{Ge}$ of C. auris-10 Ge of C. albicans; (A3) 10 Ge of C. auris-10 Ge of C. albicans; (B1) $100 \mathrm{Ge}$ of C. albicans-10 Ge of Candida duobushaemulonii; (B2) $100 \mathrm{Ge}$ of C. duobushaemulonii-10 Ge of C. albicans; (B3) $10 \mathrm{Ge}$ of C. duobushaemulonii-10 Ge of C. albicans; (C1) $100 \mathrm{Ge}$ of C. albicans-10 Ge of Candida haemulonii; (C2) $100 \mathrm{Ge}$ of C. haemulonii-10 Ge of C. albicans; (C3) $10 \mathrm{Ge}$ of C. haemulonii-10 Ge of C. albicans; (D1) $100 \mathrm{Ge}$ of C. albicans-10 Ge of Candida pseudohaemulonii; (D2) $100 \mathrm{Ge}$ of C. pseudohaemulonii-10 Ge of C. albicans; (D3) $10 \mathrm{Ge}$ of C. pseudohaemulonii-10 Ge of C. albicans.

\section{PCR conditions}

As our study had two phases of optimization (Westerdijk Fungal Biodiversity Institute, Netherlands) and assessment of performance with spiked serum samples (Division of Hygiene and Medical Microbiology/Reference Center for Aspergillus and Aspergillus Infections, Austria), hence, two kinds of master mixes and qPCR machines were used.

In the optimization phase, both DNA samples and pure colonies were subjected to qPCR assay (Applied Biosystems ${ }^{\circledR} 7500$ fast, Thermo Fisher Scientific Corporation). The latter was to check whether our assay is able to detect the signal from pure colonies and, hence, skip the tedious DNA extraction procedures. The following PCR conditions were used for the optimization phase: $10 \mu \mathrm{l}$ of Power Up SYBR Green Master Mix (A25742 Thermo Fisher Scientific Corporation), 10 pM of Auris-F, Auris-R, Pseud-F and Pseud-R, 8 pM for Haemu-F and Haemu-R, and $5 \mathrm{pM}$ for Duobu-F and Duobu-R, $1 \mu$ of DNA template (or $1 \mathrm{~mm}^{3}$ of pure colony) and Milli-Q water (Merck Millipore, Darmstadt, Germany) to reach the final volume of $20 \mu$ l. The PCR program was as following: $50^{\circ} \mathrm{C}$ for $2 \mathrm{~min}, 95^{\circ} \mathrm{C}$ for $3 \mathrm{~min}$, followed by 40 cycles of $95^{\circ} \mathrm{C}$ for $15 \mathrm{~s}$ and $64^{\circ} \mathrm{C}$ for $30 \mathrm{~s}$. Melting curves were obtained from 65 to $95^{\circ} \mathrm{C}$ with the increment of $0.5^{\circ} \mathrm{C} / \mathrm{s}$, and data analysis was carried out by 7500 software version 2.3 (Thermo Fisher Scientific Corporation).

As for the DNA of the spiked serum samples, qPCR ingredients included $10 \mu \mathrm{lof} S$ soFast ${ }^{\mathrm{TM}} \mathrm{EvaGreen}^{\circledR}$ Supermix with Low ROX (BioRad, CA, USA), 10 pM of Auris-F, Auris-R, Pseud-F and Pseud-R, 8 pM for Haemu-F and Haemu-R, and $5 \mathrm{pM}$ for Duobu-F and Duobu-R, and $8 \mu \mathrm{l}$ of DNA samples obtained from spiked serum samples in duplicate. The same PCR program (CFX96, Bio-Rad) used as it was optimized, with the exception of limiting melt curve analysis from 70 to $90^{\circ} \mathrm{C}$, and the increment of $0.1^{\circ} \mathrm{C} / \mathrm{s}$. Obtained data were analyzed by Bio-Rad CFX 

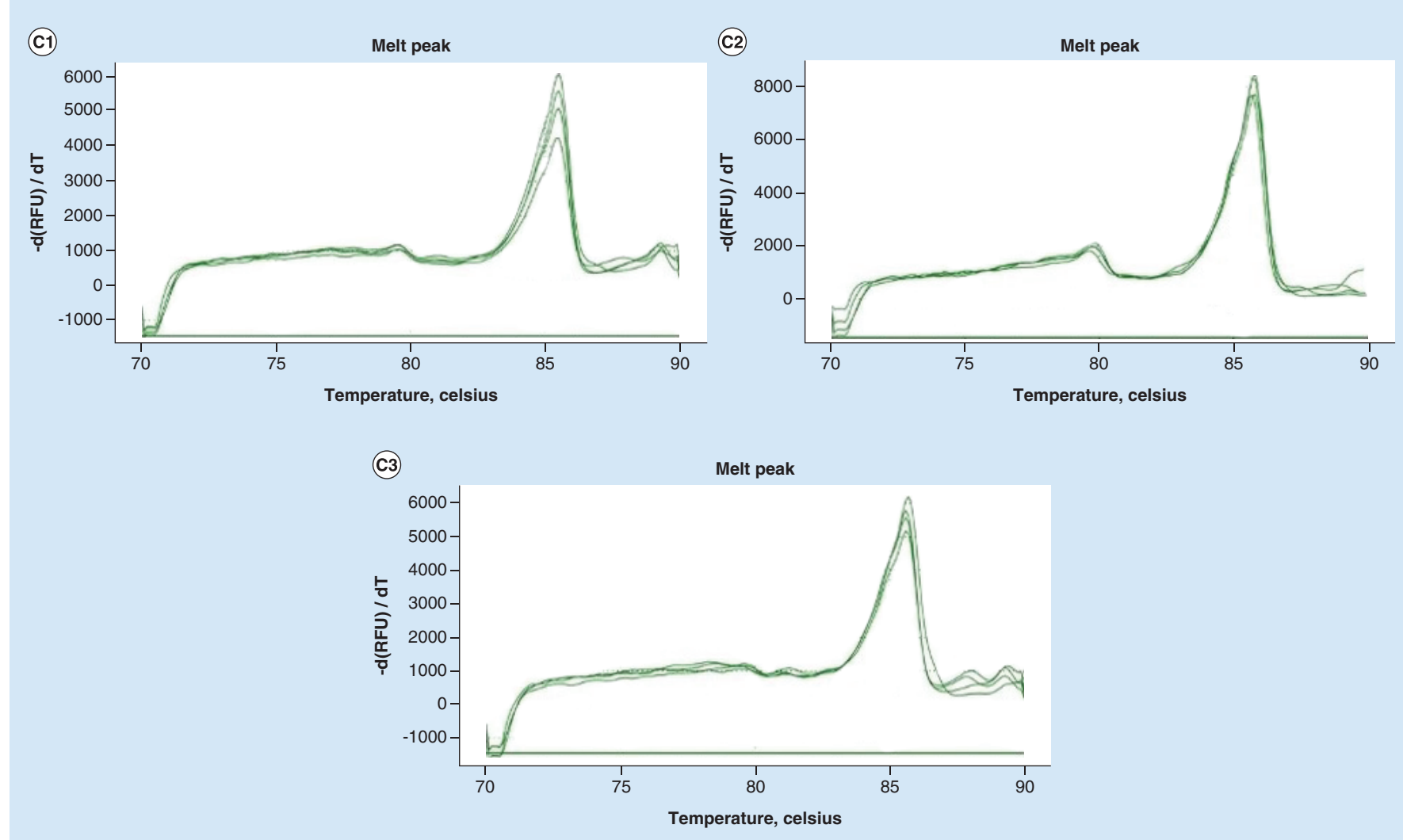

Figure 4. Melt curves obtained from serum samples spiked with mixture of Candida albicans and each target species, individually (cont.). (A1) A total of 100 genome (Ge) of Candida albicans-10 Ge of Candida auris; (A2) 100 Ge of C. auris-10 Ge of C. albicans; (A3) 10 Ge of C. auris-10 Ge of C. albicans; (B1) $100 \mathrm{Ge}$ of C. albicans-10 Ge of Candida duobushaemulonii; (B2) $100 \mathrm{Ge}$ of C. duobushaemulonii-10 Ge of C. albicans; (B3) $10 \mathrm{Ge}$ of C. duobushaemulonii-10 Ge of C. albicans; (C1) $100 \mathrm{Ge}$ of C. albicans-10 Ge of Candida haemulonii; (C2) $100 \mathrm{Ge}$ of C. haemulonii-10 Ge of C. albicans; (C3) 10 Ge of C. haemulonii-10 Ge of C. albicans; (D1) $100 \mathrm{Ge}$ of C. albicans-10 Ge of Candida pseudohaemulonii; (D2) $100 \mathrm{Ge}$ of C. pseudohaemulonii-10 Ge of C. albicans; (D3) $10 \mathrm{Ge}$ of C. pseudohaemulonii-10 Ge of C. albicans.

Manager Version 3.1 (Bio-Rad). Efficiency of utilized primers, costs and avoiding cross reaction were the most important factors ruling the concentration of primers. Candida auris as the main target species had the highest primer concentration $(10 \mathrm{pM})$, while the rest of target species due to possessing higher efficiency ( 8 and $5 \mathrm{pM})$ required less amount of respective primers.

\section{Sequencing}

Sequencing of D1/D2 domain of large subunit of rDNA was performed for all the 283 strains to explicitly approve the identity of each strain. In order to report the identity of each species, obtained sequences were subjected to BLAST program (https://blast.ncbi.nlm.nih.gov/Blast.cgi).

\section{Statistical analysis}

SPSS (version 25, International Business Machines Corporation, NY, USA) was used for statistical analysis. Results were presented as mean \pm standard deviation for normal data or median with interquartile ranges for non-normal data.

\section{Results}

Analytical sensitivity, reproducibility \& specificity

Based on the melting temperature of the target species, the designed primers unequivocally distinguished $C$. auris, C. haemulonii, C. duobushaemulonii and C. pseudohaemulonii. Melting temperatures for C. auris, C. haemulonii, C. duobushaemulonii and C. pseudohaemulonii were $84.10 \pm 0.25^{\circ} \mathrm{C}, 85.69 \pm 0.21^{\circ} \mathrm{C}, 82.44 \pm 0.25^{\circ} \mathrm{C}$ and 


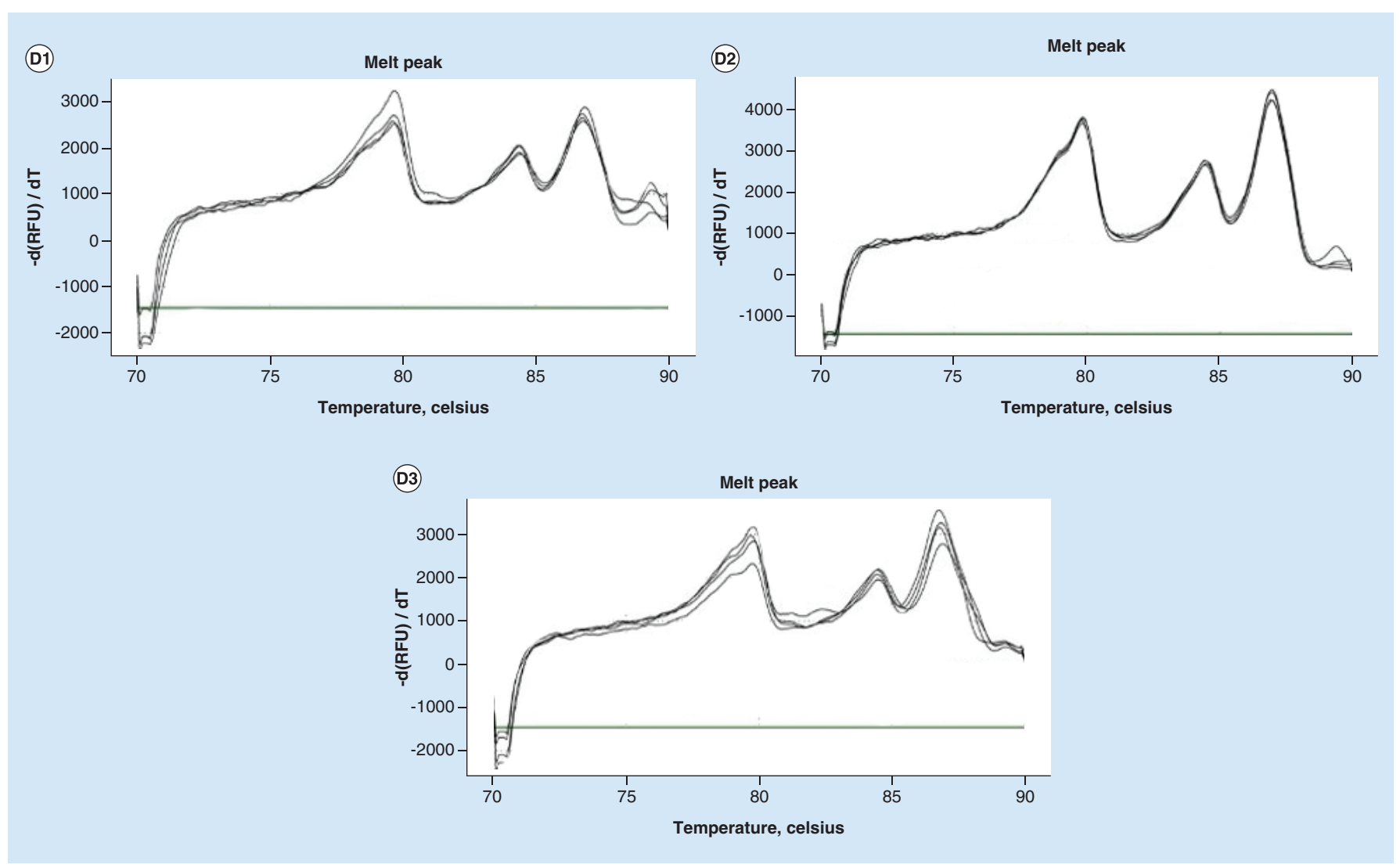

Figure 4. Melt curves obtained from serum samples spiked with mixture of Candida albicans and each target species, individually (cont.). (A1) A total of 100 genome (Ge) of Candida albicans-10 Ge of Candida auris; (A2) $100 \mathrm{Ge}$ of C. auris-10 Ge of C. albicans; (A3) 10 Ge of C. auris-10 Ge of C. albicans; (B1) $100 \mathrm{Ge}$ of C. albicans-10 Ge of Candida duobushaemulonii; (B2) $100 \mathrm{Ge}$ of C. duobushaemulonii-10 Ge of C. albicans; (B3) $10 \mathrm{Ge}$ of C. duobushaemulonii-10 Ge of C. albicans; (C1) $100 \mathrm{Ge}$ of C. albicans-10 Ge of Candida haemulonii; (C2) $100 \mathrm{Ge}$ of C. haemulonii-10 Ge of C. albicans; (C3) $10 \mathrm{Ge}$ of C. haemulonii-10 Ge of C. albicans; (D1) $100 \mathrm{Ge}$ of C. albicans-10 Ge of Candida pseudohaemulonii; (D2) $100 \mathrm{Ge}$ of C. pseudohaemulonii-10 Ge of C. albicans; (D3) $10 \mathrm{Ge}$ of C. pseudohaemulonii-10 Ge of C. albicans.

$79.16 \pm 0.18^{\circ} \mathrm{C}$, respectively (Figure $1 \mathrm{~A} \& \mathrm{D}$, Supplementary Table 4). No difference in melting temperature $(\mathrm{p}>0.05)$ was shown among C. auris strains from different countries (Figure 1C). All the target strains were correctly identified except for CBS 12371, which was wrongly designated as C. pseudohaemulonii, while our assay in accordance to D1/D2 sequencing and MALDI-TOF MS identified this strain as C. haemulonii. When the DNA samples obtained from pure cultures, the LOD for C. auris, C. pseudohaemulonii and C. duobushaemulonii was 10 $\mathrm{CFU} / \mathrm{PCR}$ with a Cq value of 32, and the LOD for C. haemulonii was $10 \mathrm{CFU} / \mathrm{PCR}$ with a Cq value of 29. The average of $\mathrm{R}^{2}$ value for six consecutive runs of qPCR was 0.99 , indicating a high degree of reproducibility of this assay (Figure 1B). Specificity tests showed no cross reactivity with the other fungal species and genomic human DNA. Subjecting 105 clinical isolates of $C$. auris from Kuwait to our qPCR gave the same results as those obtained by MALDI-TOF MS and D1/D2 domain of ribosomal large subunit (LSU) rDNA sequencing, resulting in 100\% agreement with these methods. Using blinded test set, our qPCR showed $100 \%$ accuracy and pure colonies as the pure DNA samples successfully identified target species.

\section{Performance assessment with spiked serum samples}

Results obtained from spiked serum samples revealed that our qPCR assay can reliably identify and detect ten genomes of $C$. auris $(\mathrm{Cq}=31.75)$ and one genome of $C$. haemulonii $(\mathrm{Cq}=34.82), C$. pseudohaemulonii $(\mathrm{Cq}$ $=34.82)$ and $C$. duobushaemulonii $(\mathrm{Cq}=30.61)$ (Figure $2 \mathrm{~A} \& \mathrm{C})$, and showed no cross reaction with negative serum samples (Figure 2D). Spiked serum samples with $C$. auris and $C$. haemulonii showed an identical melt curve as what was obtained for the pure DNA of both species (Figure 2A). Unexpectedly $C$. duobushaemulonii and $C$. pseudohaemulonii generated unique triple peaks specific for each species (Figure 2A). Repeated experimentation of 
spiking serum samples with $C$. pseudohaemulonii and $C$. duobushaemulonii provided the same results. However, in order to prove that these frequent melting peaks are unique for $C$. pseudohaemulonii and $C$. duobushaemulonii, $500 \mu \mathrm{l}$ of serum samples were spiked with three orders of magnitude $\left(10^{2}\right.$ genomes to $10^{0}$ genome) DNA of $C$. albicans, Candida parapsilosis, Candida glabrata, Candida tropicalis and Candida krusei (Figure 3A \& C). As it is shown in the Figure 3, serum samples spiked with the DNA of aforementioned Candida species and negative serum samples generated irregular-shaped melt curves, while melt peaks for $C$. pseudohaemulonii and $C$. duobushaemulonii consistently and efficiently resulted in the same melt curve patterns and turned out that those patterns are specific and distinctive fingerprints of these two species (Figure $3 \mathrm{~A} \& \mathrm{~B}$ ). In order to prove that frequent melting peaks were not resulted from serum background, we utilized serum samples from two healthy individuals and both serums resulted in identical and irregular melting peaks. Spiked serum samples with $C$. auris, C. haemulonii, $C$. pseudohaemulonii and $C$. duobushaemulonii showed acceptable reproducibility value $\left(\mathrm{R}^{2} \geq 0.98\right)$. These observations indicated that serum samples could be a suitable blood fraction for detection and identification of target species when using our multiplex qPCR assay.

In simulated co-infection experiment, serum samples were spiked with DNA mixture of C. albicans and each target species individually. Results from the qPCR of simulated co-infection test revealed that regardless of concentration of DNA of C. albicans (ten-times more, ten-times less and equal to target species) target species were detected and distinguished unequivocally (Figure 4).

\section{Discussion}

The lack of reliable clinical diagnostic tools potentially led to underestimation and neglection of $C$. auris cases [17,2729]. When facing $C$. auris outbreaks, microbiologists mainly handle cultures, environmental swab or clinical samples (blood, sputum, urine). Using biochemical assays such as VITEK, API20C-AUX, AuxaColor 2, BD Phoenix, MicroScan etc. $90 \%$ of the C. auris isolates are misidentified as C. haemulonii (most commonly), Candida famata, Candida sake, Candida lusitaniae, Candida catenulata, Candida guilliermondii, C. parapsilosis, C. tropicalis, C. albicans, Rhodotorula glutinis and Saccharomyces cerevisiae [5].

Detecting $C$. auris directly from clinical or environmental swab samples in a culture-independent manner is essential for outbreak control. For environmental screening purposes, Leach et al. (2017) reported the ability of single-plex probe-based qPCR for detection of C. auris from environmental swabs [18]. Although, in outbreaks environmental screening is an essential part of infection control, identification of patients suspected with bloodborne infections by $C$. auris and its relatives is of a great importance. Epidemiological studies revealed that $C$. auris can represent up to $5.2 \%$ of candidemia cases in India [30], although with a lower prevalence, fatal bloodstream infection due to this pathogen is reported from other countries [9]. Moreover, it is estimated that delay of $1 \mathrm{~h}$ in starting immediate appropriate antimicrobial therapy for ICU patients will increase the mortality rate [31]. Thus, the urge for a rapid, specific, reliable and economic molecular means of identification from clinical samples is highly relevant. Herein for the first time, we developed a multiplex PCR assay that can detect $C$. auris and its relatives directly from serum samples in a culture-independent manner. Testing our QPCR with the serum samples spiked with serially diluted DNA of target species, showed an exceptional LOD of one genome for $C$. haemulonii, $C$. pseudohaemulonii and C. duobushaemulonii, and ten genomes for $C$. auris. Moreover, serum due to its applicability for downstream applications such as serology tests and its ease of DNA extraction procedure, has earned reputation for being a suitable blood fraction for identification of fungemia [32]. It has been shown that for identification of invasive candidiasis, serum samples showed the same performance as whole blood samples when subjected to realtime PCR [33]. Additionally, serum has been regarded as a suitable matrix for validation of PCR-based assays meant to detect Candida species from infected patients [34-36]. The diagnostic strategy using our assay can dramatically shorten the clinical turn-around time (Supplementary Figure 1). Moreover, our qPCR assay utilizes the most basic and inexpensive qPCR chemistry, that is, SYBR GreenI and EVA Green, and offers the first qPCR assay with the potential clinical utility for identification of $C$. auris and its relatives. Moreover, despite the fact that $C$. albicans accounts for the majority of mixed infections, which could affect the specificity and sensitivity of PCR assays [25], our assay regardless of the DNA concentration of $C$. albicans (ten-times more, ten-times less and equal to target species) readily identified $C$. auris, $C$. haemulonii, $C$. pseudohaemulonii and $C$. duobushaemulonii in serum samples spiked the mixture DNA of target species and C. albicans. This observation reinforced the specificity of our qPCR assay in mixed infections.

For specificity testing, our assay was challenged with 111 reference strains representing 68 nontarget yeast and filamentous fungal species, while in the published melting curve-based qPCR, only 19 nontarget species were 
tested [37]. Large difference in melting temperature between $C$. auris and its relatives is another advantage of our qPCR, as PCR inhibitors, such as humic acid, melanin, hematin and collagen, are commonly found in the environmental and clinical samples and they can change the melting curve temperature causing melt temperature shift followed by misidentification [38]. In our assay, the difference in average melting temperature between $C$. auris and C. pseudohaemulonii, C. duobushaemulonii, C. haemulonii were 5, 1.7 and $1.6^{\circ} \mathrm{C}$, respectively. Additionally, the minimum difference in melting temperature between any two species was $1.1^{\circ} \mathrm{C}$. While in the published melting curve qPCR, small differences in melting temperature between $C$. auris and $C$. duobushaemulonii and $C$. haemulonii were observed $\left(0.4\right.$ and $1^{\circ} \mathrm{C}$, respectively) [37]. On the other hand, despite the presence of heterogeneity in the submitted sequences of $C$. haemulonii complex, that is, $C$. haemulonii var. vulnera and C. haemulonii (C. haemulonii Group I), our assay successfully amplified various strains within this complex [39].

Although, a given developed diagnostic assays should be assessed with the relevant clinical materials obtained from suspected patients, in our situation due to the lack of such clinical sources we had to spike serum samples with the DNA of target species. Hence, clinical performance and utility of our qPCR assay warrants further experimentations in settings where suspected patients exist.

In summary, we developed a novel multiplex qPCR, which can discriminate $C$. auris, from $C$. haemulonii, $C$. duobushaemulonii and $C$. pseudohaemulonii in one multiplex qPCR reaction. The fact is that our multiplex assay has been validated by using a comprehensive set of reference strains and clinical isolates, and proved to be rapid, specific and sensitive with spiked serum samples, indicating that it might have the applications for environmental screening and diagnostic purposes from clinical samples.

\section{Conclusion}

Culture-independent and species-level identification of the multidrug-resistant opportunistic yeast species Candida auris, C. haemulonii, C. pseudohaemulonii and C. duobushaemulonii is an important clinical practice. Involved healthcare settings of various countries can evaluate the specificity and sensitivity our multiplex qPCR assay in the light of culture as the gold standard technique. If proved to be sensitive and specific, it will show implications in early diagnosis followed by adminstartion of appropriate antifungal drugs. Moreover, its inexpensiveness, rapidity and sensitivity might highlight its applicability for environmental screening.

\section{Summary points}

- Based on the simple melt curve technique, our multiplex qPCR can distinguish and identify Candida auris, and its relatives, Candida haemulonii, Candida duobushaemulonii and Candida pseudohaemulonii.

- When DNAs obtained from pure colonies were used, the limit of detection for C. auris, C. haemulonii, C. pseudohaemulonii and C. duobushaemulonii was 10 CFU/PCR, with a high degree of reproducibility $\left(R^{2}=0.99\right)$.

- Specificity tests showed no cross reactivity with the other fungal species and genomic human DNA.

- Subjecting pure colonies of target strains showed the expected melting temperature, which indicated good compatibility of our assay when using pure colonies without DNA extraction.

- Results obtained from spiked serum samples revealed that our qPCR assay reliably identifies and detects ten genomes of $C$. auris $(C t=31.75)$ and one genome of $C$. haemulonii $(C t=34.82), C$. pseudohaemulonii $(C t=34.82)$ and C. duobushaemulonii $(\mathrm{Ct}=30.61)$.

- Spiked serum samples with both $C$. auris and C. haemulonii showed acceptable reproducibility value $\left(R^{2} \geq 0.98 \%\right)$.

- Simulated co-infection test revealed that regardless of concentration of DNA of C. albicans, target species are identified unequivocally.

- Having a high degree of sensitivity and reasonable difference in the melt profile of target species reveals the potential utility of our qPCR assay for both serum samples derived from suspected patients and environmental swab samples for infection control purposes. 
Financial \& competing interests disclosure

This work was supported by the National Health Department (2018ZX10101003), EU's Horizon 2020 Research and Innovation Program under the Marie Sklodowska-Curie grant (642095), National Natural Science Foundation of China (31770161), Second Military Medical University (2017JZ47) and Shanghai Science and Technology Committee (17DZ2270900 and 14495800500). The authors have no other relevant affiliations or financial involvement with any organization or entity with a financial interest in or financial conflict with the subject matter or materials discussed in the manuscript apart from those disclosed.

No writing assistance was utilized in the production of this manuscript.

\section{Open access}

This work is licensed under the Attribution-NonCommercial-NoDerivatives 4.0 Unported License. To view a copy of this license, visit http://creativecommons.org/licenses/by-nc-nd/4.0/

\section{References}

Papers of special note have been highlighted as: $\bullet \bullet$ of considerable interest

1. Pfaller MA, Diekema DJ, Gibbs DL et al. Results from the ARTEMIS DISK Global Antifungal Surveillance Study, 1997 to 2007 : 10.5-year analysis of susceptibilities of noncandidal yeast species to fluconazole and voriconazole determined by CLSI standardized disk diffusion testing. J. Clin. Microbiol. 47(1), 117-123 (2009).

2. Miceli MH, Diaz JA, Lee SA. Emerging opportunistic yeast infections. Lancet. Infect. Dis. 11(2), 142-151 (2011).

3. Jeffery-Smith A, Taori SK, Schelenz S et al. Candida auris: a review of the literature. Clin. Microbiol. Rev 31(1), pii: e00029-00017 (2017).

-• Misidentification through routinely used phenotypic and biochemical assays. Candida auris is easily misidentified as other yeast species when tested with the routinely used phenotypic and biochemical assays.

4. Fang W, Zhang L, Liu J et al. Tuberculosis/cryptococcosis co-infection in China between 1965 and 2016. Emerg. Microbes. Infect. 6(8), e73 (2017).

5. Chowdhary A, Sharma C, Meis JF. Candida auris: a rapidly emerging cause of hospital-acquired multidrug-resistant fungal infections globally. PLoS Pathog. 13(5), e1006290 (2017).

-. Persistent colonizer in hospitals. Candida auris for a long period of time can stay in the hospitals and clinical settings.

6. Ben-Ami R, Berman J, Novikov A et al. Multidrug-resistant Candida haemulonii and C. auris, Tel Aviv, Israel. Emerg. Infect. Dis. 23(1), 195-203 (2017).

7. Ruiz-Gaitan A, Moret AM, Tasias-Pitarch M et al. An outbreak due to Candida auris with prolonged colonisation and candidaemia in a tertiary care European hospital. Mycoses 61(7), 498-505 (2018).

8. Lockhart SR, Etienne KA, Vallabhaneni S et al. Simultaneous emergence of multidrug-resistant Candida auris on 3 continents confirmed by whole-genome sequencing and epidemiological analyses. Clin. Infect. Dis. 64(2), 134-140 (2017).

-• Multidrug resistance. Clinical isolates of $C$. auris can show multidrug-resistant nature to two or three classes of antifungals posing a threat to its treatment.

9. Osei Sekyere J. Candida auris: a systematic review and meta-analysis of current updates on an emerging multidrug-resistant pathogen. Microbiologyopen doi:10.1002/mbo3.578 (2018) (Epub ahead of print).

-• Cause of fatal outbreaks. Once established in healthcare settings, it can be easily transferred to the other hospitals in different cities, countries and even continents.

10. Kim MN, Shin JH, Sung H et al. Candida haemulonii and closely related species at 5 university hospitals in Korea: identification, antifungal susceptibility, and clinical features. Clin. Infect. Dis. 48(6), e57-e61 (2009).

11. Hou X, Xiao M, Chen SC et al. Identification and antifungal susceptibility profiles of Candida haemulonii species complex clinical isolates from a multicenter study in China. J. Clin. Microbiol. 54(11), 2676-2680 (2016).

12. Khan ZU, Al-Sweih NA, Ahmad S et al. Outbreak of fungemia among neonates caused by Candida haemulonii resistant to amphotericin B, itraconazole, and fluconazole. J. Clin. Microbiol. 45(6), 2025-2027 (2007).

13. Ramos R, Caceres DH, Perez M et al. Emerging multidrug-resistant Candida duobushaemulonii infections in Panama hospitals: importance of laboratory surveillance and accurate identification. J. Clin. Microbiol. 56(7), (2018).

-. Wrong identification and overestimation in number of $C$. auris. By using reliable means of identification wrongly identified $C$. auris isolates were identified as Candida duobushaemulonii leading to revealing of the genuine epidemiology of this species.

14. Sugita T, Takashima M, Poonwan N, Mekha N. Candida pseudohaemulonii Sp. Nov., an amphotericin B-and azole-resistant yeast species, isolated from the blood of a patient from Thailand. Microbiol. Immunol. 50(6), 469-473 (2006).

-. Multidrug resistance nature of its siblings. Species within the Candida baemulonii complex, including Candida haemulonii, Candida pseudohaemulonii and Candida duobushaemulonii are multidrug-resistant emerging yeast species that are closely related to $C$. auris. References 10-14 
15. Lepak AJ, Zhao M, Berkow EL, Lockhart SR, Andes DR. Pharmacodynamic optimization for treatment of invasive Candida auris infection. Antimicrob. Agents Chemother. 61(8), 1-5 (2017).

16. Fakhim $\mathrm{H}$, Chowdhary A. In vitro interactions of echinocandins with triazoles against multidrug-resistant Candida auris. Antimicrob. Agents Chemother. 61(11), pii: e01056-17 (2017).

17. Mizusawa M, Miller H, Green R et al. Can multidrug-resistant Candida auris be reliably identified in clinical microbiology laboratories? J. Clin. Microbiol. 55(2), 638-640 (2017).

18. Leach L, Zhu Y, Chaturvedi S. Development and validation of a real-time PCR assay for rapid detection of Candida auris from surveillance samples. J. Clin. Microbiol. 56(2), pii: e01223-17 (2018).

19. Arastehfar A, Fang W, Badali H et al. Low-cost tetraplex PCR for the global spreading multi-drug resistant fungus, Candida auris and its phylogenetic relatives. Front. Microbiol. 9,1119 (2018).

20. Voelker R. New test identifies Candida auris. JAMA 319(21), 2164 (2018).

21. Snayd M, Dias F, Ryan RW, Clout D, Banach DB. Misidentification of Candida auris by RapID Yeast Plus, a commercial, biochemical enzyme-based manual rapid identification system. J. Clin. Microbiol. 56(5), 1-2 (2018).

22. Vlek A, Kolecka A, Khayhan $\mathrm{K}$ et al. Interlaboratory comparison of sample preparation methods, database expansions, and cutoff values for identification of yeasts by matrix-assisted laser desorption ionization-time of flight mass spectrometry using a yeast test panel. J. Clin Microbiol. 52(8), 3023-3029 (2014).

23. Khan Z, Ahmad S, Benwan K et al. Invasive Candida auris infections in Kuwait hospitals: epidemiology, antifungal treatment and outcome. Infection 46(5), 641-650 (2018).

-. High mortality rate. The rate of mortality for C. auris can go to up to $60 \%$.

24. Loeffler J, Henke $\mathrm{N}$, Hebart $\mathrm{H}$ et al. Quantification of fungal DNA by using fluorescence resonance energy transfer and the light cycler system. J. Clin. Microbiol. 38(2), 586-590 (2000).

25. Jensen J, Munoz P, Guinea J, Rodriguez-Creixems M, Pelaez T, Bouza E. Mixed fungemia: incidence, risk factors, and mortality in a general hospital. Clin. Infect. Dis. 44(12), e109-e114 (2007).

26. Diaz MR, Boekhout T, Kiesling T, Fell JW. Comparative analysis of the intergenic spacer regions and population structure of the species complex of the pathogenic yeast Cryptococcus neoformans. FEMS Yeast Res. 5(12), 1129-1140 (2005).

27. Chowdhary A, Sharma C, Duggal S et al. New clonal strain of Candida auris, Delhi, India. Emerg. Infect. Dis. 19(10), 1670-1673 (2013).

28. Kathuria S, Singh PK, Sharma C et al. Multidrug-resistant Candida auris misidentified as Candida haemulonii: characterization by matrix-assisted laser desorption ionization-time of flight mass spectrometry and DNA sequencing and its antifungal susceptibility profile variability by Vitek 2, CLSI Broth Microdilution, and Etest Method. J. Clin. Microbiol. 53(6), 1823-1830 (2015).

29. Centers for Disease Control and Prevention. Clinical alert to US healthcare facilities - June 2016: global emergence of invasive infections caused by the multidrug-resistant yeast Candida auris. Centers for Disease Control and Prevention, Atlanta, GA, USA (2016). www.cdc.gov/fungal/diseases/candidiasis/candida-auris-alert.html

30. Chakrabarti A, Sood P, Rudramurthy SM et al. Incidence, characteristics and outcome of ICU-acquired candidemia in India. Intens. Care Med. 41(2), 285-295 (2015).

31. Kumar A, Roberts D, Wood KE et al. Duration of hypotension before initiation of effective antimicrobial therapy is the critical determinant of survival in human septic shock. Crit. Care Med. 34(6), 1589-1596 (2006).

32. White PL, Mengoli C, Bretagne S et al. Evaluation of Aspergillus PCR protocols for testing serum specimens. J. Clin. Microbiol. 49(11), 3842-3848 (2011).

33. Fortun J, Meije Y, Buitrago MJ et al. Clinical validation of a multiplex real-time PCR assay for detection of invasive candidiasis in intensive care unit patients. J. Antimicrob. Chemoth. 69(11), 3134-3141 (2014).

34. Dunyach C, Bertout S, Phelipeau C, Drakulovski P, Reynes J, Mallie M. Detection and identification of Candida spp. in human serum by LightCycler real-time polymerase chain reaction. Diagn. Micr. Infec. Dis. 60(3), 263-271 (2008).

35. Ahmad S, Khan Z, Mustafa AS, Khan ZU. Seminested PCR for diagnosis of candidemia: comparison with culture, antigen detection, and biochemical methods for species identification. J. Clin. Microbiol. 40(7), 2483-2489 (2002).

36. Mcmullan R, Metwally L, Coyle PV et al. A prospective clinical trial of a real-time polymerase chain reaction assay for the diagnosis of candidemia in nonneutropenic, critically ill adults. Clin. Infect. Dis. 46(6), 890-896 (2008).

37. Kordalewska M, Zhao Y, Lockhart SR, Chowdhary A, Berrio I, Perlin DS. Rapid and accurate molecular identification of the emerging multidrug resistant pathogen Candida auris. J. Clin. Microbiol. 55(8), 2445-2452 (2017).

38. Mccord B, Opel K, Funes M, Zoppis S, Jantz LM. An investigation of the effect of DNA degradation and inhibition on PCR amplification of single source and mixed forensic samples. (2011). www.ncjrs.gov/pdffiles1/nij/grants/236692.pdf

39. Cendejas-Bueno E, Kolecka A, Alastruey-Izquierdo A et al. Reclassification of the Candida haemulonii complex as Candida haemulonii (C. haemulonii group I), C. duobushaemulonii sp. nov. (C. haemulonii group II), and C. haemulonii var. vulnera var. nov.: three multiresistant human pathogenic yeasts. J. Clin. Microbiol. 50(11), 3641-3651 (2012). 